Volume 2

Number 1 Volume 2, Number 1 (March 2009)

Article 3

2009

\title{
L'Acte de Juger entre Connaissance et Distance du Monde
}

Louis LeBel

Follow this and additional works at: http:// digitalcommons.osgoode.yorku.ca/ohrlp

\section{Citation Information}

LeBel, Louis. "L’Acte de Juger entre Connaissance et Distance du Monde." Osgoode Hall Review of Law and Policy 2.1 (2014): 29-40. http://digitalcommons.osgoode.yorku.ca/ohrlp/vol2/iss1/3

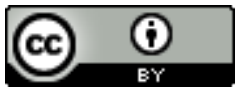

This work is licensed under a Creative Commons Attribution-NonCommercial-NoDerivs 3.0 Unported License.

This Lecture is brought to you for free and open access by the Journals at Osgoode Digital Commons. It has been accepted for inclusion in Osgoode Hall Review of Law and Policy by an authorized administrator of Osgoode Digital Commons. 


\title{
LECTURE
}

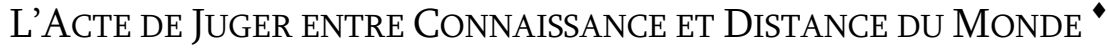

\author{
The Honourable Louis LeBel ${ }^{*}$
}

In this lecture, the author addresses the appropriate role of the judge in society. Judicial independence and impartiality require that a judge seek to distance herself from the world, prior connections, conflicts of interest and social activism. Although judges may take judicial notice of certain facts, they are otherwise limited to the arguments of the parties to a dispute. Nevertheless, judges must be attentive and critical observers of the world, eager to learn and gain knowledge about the evolving nature of economic life, science, religion and all other issues in contemporary society because distance and impartiality from the world do not imply ignorance of it.

\section{TABLE OF CONTENTS}

I. INTRODUCTION 29

II. Distance Du MONDE

A. DÉONTOLOGIE, INDÉPENDANCE ET IGNORANCE 31

B. LA PROCÉDURE CIVILE ET L'IGNORANCE JUDICIAIRE 33

C. LE DEVOIR DE CULTURE DU JUGE 35

\section{INTRODUCTION}

Le Congrès de la magistrature de 2008 s'interroge sur la figure du juge dans la société. Il examine non seulement l'image du juge, mais aussi la réalité du métier de magistrat. Quelles sont les attentes de la société ? Comment les satisfaire en respectant les contraintes propres à l'acte de juger, notamment ses obligations de réserve et de neutralité ? Comment saurait-il être présent au monde et éloigné de celui-ci, informé de son univers mais tenu dans une ignorance strictement aménagée de celui-ci ? Comment résoudre cette aporie?

Mes commentaires examineront comment certains aspects de ces problèmes se posent dans l'exécution de l'acte de juger. Il s'agit

- (C) 2009 The Honourable Louis LeBel, Justice of the Supreme Court of Canada.

* The Honourable Louis LeBel, Justice of the Supreme Court of Canada. The Justice's remarks were delivered at the Congrés de la Magistrature, Québec City, November 2008. An English version of this lecture should appear in a subsequent issue of the Review. 
sans aucun doute de l'acte le plus caractéristique de la profession judiciaire. Dire le droit, départager des prétentions contradictoires et déterminer les faits entre le vrai et le faux constituent le cœur même de l'activité du juge. On peut être juge-médiateur, conciliateur, administrateur ou parfois agent de l'État. Cependant, à un moment ou l'autre de sa carrière, le juge doit affronter les difficultés de l'acte de jugement. Les règles qui gouvernent la vie du juge veulent encadrer cet acte de jugement, pour assurer l'intégrité de celui-ci.

Les symboles traditionnels de la justice illustrent à la fois les perceptions sociales du rôle du juge et les contraintes de la profession judiciaire. Tantôt la justice porte des balances égales : on la veut juste au sens le plus étroit du terme, c'est-à-dire capable de conserver l'égalité entre les parties, sans parti pris et sans faveur. Parfois, on la sculpte le glaive à la main, sans peur et efficace, prête à trancher de façon décisive, une fois que les plateaux de la balance se sont inclinés. Enfin, il arrive qu'on peigne une justice aveuglée, un bandeau sur les yeux, mais tenant toujours la balance égale.

La cécité et l'ignorance seraient-elles alors les conditions nécessaires de la justice de l'acte de juger du maintien de l'équilibre des plateaux de la balance ? L’ignorance, protégée par l'aveuglement constituerait-elle une garantie nécessaire de l'acte de juger? La nature des règles de déontologie et les structures fondamentales du procès civil et pénal peuvent le suggérer. Pourtant, la nécessité de la connaissance et du souci du monde ne reconnaît-elle pas la présence d'une obligation de culture et d'initiative pour que l'acte de juger demeure pertinent et efficace dans la vie de la société ? L'indépendance judiciaire ne saurait reposer sur l'ignorance, la passivité et la crainte du changement. L'éthique de la distance du monde ne doit pas dériver, même inconsciemment, vers l'acceptation de l'ignorance de l'univers et de la société. 


\section{DISTANCE DU MONDE}

\section{A. DÉONTOLOGIE, INDÉPENDANCE ET IGNORANCE}

Le Canada appartient à la famille des démocraties libérales. Parmi les éléments fondamentaux des structures politiques de ce type de société se trouve une justice indépendante destinée à assurer le respect du principe de la soumission de tous à la loi, le " rule of law ", dans le vocabulaire de la common law. Si l'indépendance de cette justice relève de l'ordre constitutionnel, elle se fonde aussi sur une tradition qui exige la présence d'une personne neutre et pour cela impartiale et indépendante afin de régler les conflits sur les droits et obligations des membres de la société. L’influence de cette tradition crée des attentes qui exigent l'adoption de comportements compatibles avec la neutralité de ce tiers impartial, le juge. La préservation de cette neutralité semble impliquer la création d'un voile ou d'un écran qui sépare le juge du monde ambiant pour assurer sa capacité d'écoute et l'intégrité de son pouvoir de décision. Cette volonté inspire les règles de déontologie régissant la vie et le travail des juges. Cet encadrement déontologique peut même créer la perception que l'indépendance judiciaire repose sur l'absence et l'ignorance du monde.

Je tire un exemple de cet encadrement du Code de déontologie de la Magistrature ${ }^{1}$ qui s'applique aux juges nommés par la province de Québec. Ce code, conçu en termes très généraux, comporte des éléments qui invitent à conclure à un devoir d'ignorance et de distance. Selon ses dispositions, le juge «... doit de façon manifeste être impartial et objectif $»^{2}$. Il doit être et paraître impartial et objectif. Pour ce faire, il lui faut se garder des conflits d'intérêts, donc se dégager des situations où son détachement serait compromis ou semblerait l'être ${ }^{3}$. L'article 6 lui interdit d'ailleurs les activités incompatibles avec la fonction judiciaire, qui le feraient " déroger » aux yeux du public. La sérénité et la réserve s'imposent à lui. Le Code de déontologie dessine l'image du magistrat éloigné des turbulences du

\footnotetext{
${ }^{1}$ Code de déontologie de la Magistrature, ch. T.-16, r. 4.1.

${ }^{2}$ Code de déontologie, art. 5.

${ }^{3}$ Code de déontologie, art. 4.
} 
monde. Pour conserver sa sérénité, il se situe à côté de celui-ci et non en lui. Cette vision est répandue au Canada. Par exemple, elle est aussi celle des principes généraux de déontologie recommandés par le Conseil de la magistrature du Canada, qui insistent fortement sur la nécessité par le juge de prendre ses distances à l'égard de la société ambiante ${ }^{4}$.

Ces règles cherchent à protéger le magistrat et sa fonction. Codifiées ou laissées à la tradition, elles demeurent toutefois au même effet, dans leur volonté d'assurer la neutralité du magistrat par son éloignement de la société ambiante. Des critiques prétendraient qu'elles le placent peut être dans une bulle, qui le prive d'un contact suffisant avec l'air du temps.

En effet, le magistrat, avant tout, prend ses distances à l'égard des débats sociaux. S'il s'engage, l'opinion publique lui reprochera de se départir de sa réserve. Les règles déontologiques lui feront grief de son engagement. Le juge ne peut se transformer en un activiste social ${ }^{5}$. On s'inquiète même des activités de son conjoint ${ }^{6}$. On ne lui permet pas non plus d'être homme ou femme d'affaire. D'ailleurs, la question des investissements du juge demeure délicate. On se demande parfois s'il ne devrait pas abandonner toute activité d'investissement direct pour confier la gestion de son patrimoine à des tiers ou à des fiduciaires.

Les activités intellectuelles du juge deviennent même parfois suspectes. Peut-il écrire? Peut-il collaborer à la littérature juridique ? Est-il autorisé à enseigner ses propres opinions sur les questions diverses ou à s'exprimer à ce sujet ? Des attitudes différentes ont été adoptées. J'ai parfois entendu dire que, dans le passé, des juges en chef avaient pris la position très ferme que leurs juges devaient réserver leurs plumes à la seule rédaction de leurs jugements. Cela ne fut pas mon cas, mais des collègues semblent avoir vécu des expériences différentes, selon quelques confidences. N'oublions pas non plus la nécessité de la rupture avec les anciens clients, les associations et même certaines activités caritatives. Par exemple, la déontologie

\footnotetext{
${ }^{4}$ Conseil canadien de la Magistrature : Principes de déontologie judiciaire, Ottawa, 2004, ch. 6.

${ }^{5}$ Voir Ruffo c. Le Conseil de la magistrature du Québec, 2005 QCCA 1197, par. 59 et 60.

${ }^{6}$ Re Pinochet, (1999) 2 W.L.R. 272.
} 
contemporaine déconseille fortement aux juges de participer à des activités de levée de fonds, autres que celles qui se déroulent à l'intérieur de la magistrature. De plus, aujourd'hui, l'engagement dans certaines formes d'activités sociales, dans des organismes sans but lucratif, comme les collèges ou les institutions hospitalières, est fortement découragé, notamment en raison des conflits dans lesquels peuvent parfois être engagés avec l'État, leur propre personnel ou des tiers. On n'imagine pas non plus un juge en activité chroniqueur dans les médias. Cette approche déontologique laisse donc le juge dans un isolement potentiel. Indépendant et présumément impartial, parce qu'à l'abri des influences extérieures, il doit être prêt à écouter ce qu'on lui propose, mais non à se faire l'écho de ses propres suggestions.

\section{B. LA PROCÉDURE CIVILE ET L'IGNORANCE JUDICIAIRE}

Les règles de procédure civile, que j'examinerai principalement dans la perspective du droit en vigueur au Québec, reflètent aussi la tradition juridique qu'expriment les principes déontologiques. Elles veulent circonscrire la connaissance judiciaire. Elles entendent aménager le bon usage de l'ignorance judiciaire.

Deux traits caractérisent l'attitude du droit de la preuve et de la procédure en cette matière : limites imposées à la connaissance judiciaire et restrictions aux initiatives du juge destinées à accroître sa connaissance des faits d'un dossier. Ces traits confirment la volonté que le juge conserve son impartialité, parce qu'il n'a pu se créer des opinions pré-conçues sur une affaire et sur ces problèmes. L'intégrité de sa capacité de décision serait assurée parce que le droit lui impose un devoir d'ignorer certaines questions et de s'en remettre au plaideur pour s'en instruire, en règle générale.

Ce constat s'infère du contenu même des règles qui structurent la connaissance judiciaire. Certes, l'article 2806 C.c.Q. prévoit que " nul n'est tenu de prouver ce dont le tribunal est tenu de prendre connaissance ». L'article 2809 C.C.Q. dispose par ailleurs que « le tribunal doit prendre connaissance d'office de tous faits dont la notoriété rend l'existence raisonnablement incontestable ». La connaissance d'office ou connaissance judiciaire comporte donc deux principes. Le premier exige que le juge prenne connaissance de certains faits notoires. Elle présume même qu'il les connaît. Par ailleurs, s'il ne lui interdit pas de connaître d'autres faits, le second 
principe lui commande de se comporter comme s'il les ignorait. Il lui défend, en effet, de se conduire comme s'il connaissait des faits pertinents à un litige, en dépit de la connaissance effective qu'il en possède. Je doute toutefois que cette interdiction ait des effets pratiques. La connaissance personnelle du juge de la géographie d'un lieu ou des us et coutumes d'un milieu aura son effet sur le dossier, d'une manière ou d'une autre. Cependant, au moins au niveau théorique, ce second principe élève implicitement l'ignorance même artificielle au niveau d'une garantie de l'impartialité judiciaire.

La règle reflète une méfiance profondément ancrée vis-à-vis de la subjectivité judiciaire. Elle cherche à préserver une capacité d'écoute comprise comme une vertu d'abord passive. Certes, le juge doit posséder certes une connaissance des faits pertinents pour rendre sa décision. On lui permet - on l'y oblige même - de connaître des faits notoires. On pourrait discuter longuement de la nature de ces derniers. Cependant, au delà de cette base, le juge dépend en principe des parties pour sa connaissance du dossier.

L'organisation de la procédure civile au Québec renforce la portée de cette règle. Ainsi, aux articles 290 à 292 du Code de procédure civile, elle affirme en substance que le procès appartient d'abord aux parties et à leurs avocats. Ceux-ci définissent le litige et son cadre factuel. Ils établissent ainsi l'étendue potentielle de la connaissance judiciaire. La procédure civile ne nie pas toute possibilité d'intervention judiciaire. L'article 292 C.p.c. se montre soucieux de laisser au magistrat des possibilités d'initiatives et lui fait même obligation de chercher à prévenir les injustices. Mais en définitive, bien qu'il engage l'autorité publique et fasse largement appel à ses services, et en dépit de l'élargissement des pouvoirs d'intervention du juge, le procès appartient toujours aux parties. Dans un système judiciaire fort différent de celui du Canada, la Cour de cassation de France l'a récemment souligné ${ }^{7}$.

En appel, par exemple devant la Cour suprême du Canada, la même attitude fondamentale prévaut. Il existe sans doute des exceptions permettant de présenter des preuves nouvelles. Toutefois, la Cour suprême juge en principe sur la base des faits établis par les parties. Elle s'interdit d'ailleurs de soulever d'office des moyens qui

\footnotetext{
${ }^{7}$ Assemblée plénière, 21 décembre 2007, Cour de cassation de France, Bulletin d'information, 15 avril 2008, p. 19.
} 
seraient dépourvus de base factuelle dans le dossier d'appel. La nature de son action se trouve ainsi souvent définie par le cadre processuel adopté par les parties ${ }^{8}$.

Cette attitude est encore plus clairement imposée en droit pénal. Le procès appartient au ministère public et à la défense. Le juge ne connaît officiellement que la preuve présentée par les parties. Lorsqu'un jury est constitué, l'exclusion des preuves jugées inadmissibles impose souvent aux jurés l'ignorance d'éléments parfois importants de la situation de faits dont il est saisi. Le juge siégeant seul, doit aussi tenter d'oublier les preuves qu'il rejette. L'exercice s'avère délicat. Est-il toujours réaliste ? Je ne saurais l'affirmer trop fermement. Cependant, son caractère obligatoire confirme les limites de la connaissance judiciaire et l'importance de l'ignorance judiciaire. L'existence de cette dernière s'inscrit donc fermement dans cette volonté de distance sinon d'ignorance du monde que je soulignais plus haut. Toutefois, ne nous concentrons-nous pas trop sur les règles de protection de cette indépendance et impartialité judiciaire? Ne nous arrêtons pas trop à ces seules obligations. Ne négligeons-nous pas l'importance d'obligations d'information du magistrat qui conditionneraient le bon fonctionnement des règles de la connaissance judiciaire ? Ne faut-il pas reconnaître l'existence d'un devoir de culture personnelle du juge, d'une obligation d'attention au monde et de connaissance de celui-ci et explorer leur nature?

\section{Le Devoir De Culture Du Juge}

La déontologie judiciaire s'est peu arrêtée au contenu des obligations positives du magistrat à l'égard du développement de ses connaissances et encore moins de celui de sa culture générale. Pourtant, jusqu'à quel point serait utile un magistrat qui ignore tout du monde où vivent ses contemporains ? Peut-il planer comme un extra-terrestre au-dessus de l'univers, où pourtant il continue à vivre et n'y descendre que pour témoigner de sa sagesse en prononçant ses jugements ? Au fond, peut-il être sage, s'il ne connaît pas son environnement, s'il perd conscience de ce qu'il est?

${ }^{8}$ Loi sur la Cour suprême, L.R., 1985, ch. S-26, art. 62; voir aussi B. Crane et S. Brown, Supreme Court of Canada Practice, Thomson Carswell, Toronto, 2008, p. 129 ss. 
Les codes ou règles de déontologie restent fort concis au sujet de cette question. Par exemple, le Code de déontologie des juges de la Cour du Québec affirme : " que le juge a l'obligation de maintenir sa compétence professionnelle ». Les " Principes de déontologie judiciaire " publiés par le Conseil canadien de la magistrature se montrent un peu plus conscients de cet aspect des devoirs du juge. Ainsi, au chapitre 4, traitant de la diligence du juge, l'art. 2 recommande un principe de développement des connaissances et compétences du magistrat.

"Les juges prennent les mesures qui s'imposent pour préserver et accroître les connaissances, les compétences et les qualités personnelles qui sont nécessaires à l'exercice de leurs fonctions judiciaires ».

$\mathrm{Au}$ chapitre 5, sur le droit à l'égalité, les commentaires constatent la présence d'un devoir d'information sur les diversités de la société contemporaine, ajoutent une mise en garde à l'égard des préjugés communs et insistent sur l'importance de l'attention aux changements dans la société ${ }^{9}$.

Cependant, l'obligation de diligence du juge demeure surtout comprise comme un devoir de connaître le droit et les méthodes de conduite du procès et de gestion des affaires judiciaires. Son exécution cause aujourd'hui, à mon avis, assez peu de problèmes. Selon mon expérience, les membres de la magistrature se montrent soucieux de préserver de développer leurs connaissances du droit. Des ressources parfois importantes sont mises à leurs dispositions pour ces fins. Les activités de l'Institut national de la magistrature du Canada et la participation des juges aux colloques organisés par les différentes cours en témoignent. Les pressions du travail et le manque de temps peuvent certes les gêner dans ces activités. Malgré ces obstacles, il demeure que cette obligation est bien comprise et exécutée avec diligence.

Toutefois, le problème de la compétence et de la diligence du juge se pose en termes plus larges. Je l'examine maintenant dans la perspective d'une magistrature dont les membres, comme ceux de la

${ }^{9}$ Principes, commentaires, pp. 24 et 25. 
magistrature canadienne, accèdent pour la plupart à la fonction judiciaire entre 40 ou 50 ans, après avoir vécu dans le monde juridique depuis leur sortie de l'Université pendant 15, 20, 25 ans. À ce moment, après leur nomination, ils vivent une rupture nécessaire avec les réseaux à l'intérieur desquels ils étaient intégrés, pour établir la distance nécessaire avec leurs anciens clients et leurs domaines d'activités. Ils cessent fréquemment de voir des groupes avec lesquels ils ont travaillé longtemps. S'ils rencontrent les personnes avec lesquelles ils collaboraient, leurs échanges deviennent marqués par la prudence et la réticence. Ils perdent nécessairement des sources importantes d'information au sujet du cours des affaires de la société. Les règles déontologiques que j'ai évoquées plus haut restreignent leurs activités sociales ou caritatives. Lorsque j’ai été nommé à la Cour d'appel du Québec, j'ai eu alors l'impression, après quelques mois, sinon de ne plus rien savoir, du moins d'éprouver des difficultés à suivre l'évolution de la société et encore plus celle de certains milieux économiques dans lesquels je travaillais. J'ai éprouvé le sentiment de vivre une sorte de coupure du monde. D'autres ont sans doute fait la même expérience. Au Barreau, on moque parfois gentiment ce passage en le décrivant comme l'entrée au monastère.

J'exprimerais quelques réserves à propos de ce qualificatif. Son usage témoigne toutefois d'un risque, celui d'un décrochage du juge vis-à-vis de la société ambiante. Ce décrochage entraîne le danger de concevoir le système juridique comme un ensemble clos, alors qu'il s'insère profondément dans toute la vie de la société.

Il importe alors de bien comprendre l'importance de l'obligation pour le juge de maintenir sa compétence que reconnaissent les principes de déontologie. Il ne s'agit pas seulement de la préservation de sa connaissance de droit, si importante qu'elle soit. Elle exprime un devoir d'ouverture au monde et de culture personnelle qui va au delà du seul développement des connaissances juridiques. Elle invite aussi, au refus de la passivité et de la paresse dans la conduite des affaires judiciaires. Le juge ne peut plus être un acteur dans la société sauf par son action dans le domaine judiciaire. Il doit cependant demeurer un observateur attentif de celle-ci. Pour remplir ce rôle, il doit demeurer attentif et soucieux de s'informer. Je ne parle pas ici d'une obligation d'encyclopédisme. Le temps où l'être humain pouvait prétendre que rien dans le domaine des connaissances ne lui était étranger est révolu depuis longtemps. L'étendue des 
connaissances humaines ainsi que la diversité et l'abondance des informations nous rendent nécessairement conscients de notre ignorance. On ne s'attend pas à ce que les juges se transforment en érudits, qu'ils doivent être à la fois philosophes, mathématiciens, critiques littéraires, économistes, agronomes, etc. Nous éprouvons déjà suffisamment de difficultés à être de bons juristes. Toutefois, la culture générale demeure aussi importante aujourd'hui qu'au moment où en 1930, le grand juge américain Learned Hand soulignait le rôle fondamental de disciplines comme l'histoire, la philosophie ou la littérature pour le juge dans l'exercice de sa profession ${ }^{10}$ :

... I venture to believe that it is as important to a judge called upon to pass on a question of constitutional law, to have at least a bowing acquaintance with Acton and Maitland, with Thucydides, Gibbon and Carlyle, with Homer, Dante, Shakespeare and Milton, with Machiavelli, Montaigne and Rabelais, with Plato, Bacon, Hume and Kant, as with the books which have been specifically written on the subject. For in such matters everything turns upon the spirit in which he approaches the questions before him. The words he must construe are empty vessels into which he can pour nearly anything he will. Men do not gather figs of thistles, nor supple institutions from judges whose outlook is limited by parish or class. They must be aware that there are before them more than verbal problems; more than final solutions cast in generalizations of universal applicability. They must be aware of the changing social tensions in every society which makes it an organism; which demand new schemata of adaptation; which will disrupt it, if rigidly confined.

Le contenu de la culture varie avec le temps. L'approfondissement de l'esprit et la capacité d'attention qu'elle développe demeurent. Elle est mémoire du passé, conscience du présent et vision de l'avenir. Il faut savoir ce qui se passe dans la société. On doit écouter, observer, s’informer suffisamment pour percevoir les tendances sociales et les courants culturels. On doit aussi

${ }^{10}$ Hon. Learned Hand, Sources of Tolerance, (1930) 79 University of Pennsylvania Law Review 1, p. 12. 
prêter plus d'attention à des domaines qui sont parfois proches de la vie judiciaire en raison de leurs impacts sur les affaires dont nous sommes saisis. J'en évoquerai quelques-uns. Par exemple, dans le domaine de la vie économique, il est nécessaire de comprendre les courants mondiaux, les difficultés de l'économie et leurs répercussions sur la pratique du droit rattachée aux affaires. Les difficultés que rencontrent bien des juges dans le domaine scientifique sont connues ${ }^{11}$. Dans notre pays, sauf dans des juridictions un peu spécialisées, combien de juges connaissent l'évolution des sciences et les problèmes qu'elle soulève ? Sans se plonger à fond dans le domaine, un juge doit rester au fait des questions qui se posent dans ce domaine, de leur existence, au moins à un niveau de culture générale. Ainsi, quelles que soient nos convictions personnelles, nous ne saurions non plus ignorer l'importance culturelle des religions et leur influence contemporaine sur les problèmes de la société et du droit.

Le même besoin de connaissance peut se refléter quelques fois de façon plus pratique dans la vie quotidienne du juge. La montée de l'informatique devrait nous rendre conscient de l'importance de ce domaine. Si nous ne comprenons pas l'ampleur des changements qu'elle provoque, nous serons peut être incapables de saisir la portée de leurs effets sur le fonctionnement de certaines règles de procédures. Par exemple, nous oublierons peut être, à titre d'exemple, que les examens au préalable visant une information conservée sur une base essentiellement informatique, peuvent poser des problèmes fort différents de ceux des interrogatoires et des recherches d'information que nous connaissions, il y a 30 ou 40 ans.

Il faut demeurer intéressé aux grands courants de l'évolution de la vie de la société pour éviter des ruptures entre le droit et le tissu social auquel il s'applique. Il est possible que certains des problèmes vécus par le droit familial au Canada, à partir de 1970, après l'entrée en vigueur de la législation fédérale sur le divorce, aient pu découler, en partie au moins, de l'ignorance, de la part d'un certain nombre de magistrats, des conditions réelles de la vie des familles et de l'impact des changements sociaux dans ce domaine sur leur organisation et leur fonctionnement, particulièrement à propos du rôle des femmes.

${ }^{11}$ I. Binnie, Science in the Courtroom: The mouse that roared, (2008) Advocates Society Journal, September, p. 11. 
Juges canadiens, nous avons connu les difficultés et les défis d'une génération et nous accédons à la magistrature à un moment où apparaissent les problèmes de couches nouvelles de la société. Il faut alors chercher à devenir attentif à l'apparition de ces problèmes et rester constamment en alerte. On évitera de s'attacher seulement à l'examen du monde que l'on a connu, mais on cherchera à deviner les traits de l'univers qui apparaîtra.

En même temps, cette capacité de recherche doit demeurer critique. Il ne faut pas oublier que le métier de juge repose aussi sur l'exercice d'un sens critique nécessaire à la préservation de liberté de jugement. Il ne s'agit pas ici d'absorber toutes les modes intellectuelles de temps ou d'adopter toutes les théories sociales de l'heure avec une foi aveugle. Au contraire, la connaissance que l'on conserve ou développe d'un milieu et de son histoire permet de conserver davantage la distance critique et l'impartialité nécessaire à la bonne exécution de la fonction judiciaire.

Ainsi, je suis persuadé que l'obligation de protection de la compétence professionnelle n'est pas un devoir étroit. Elle constitue une obligation large de conscience, d'attention et d'activité. Ultimement, elle permettra au juge de mieux remplir sa fonction et de prendre les initiatives nécessaires parfois dans l'exécution de sa tâche. Elle évitera au juge de se concevoir comme un instrument passif de la justice. Ce temps est fini, s'il a jamais existé. Les changements nous ont conduit ailleurs. Les méthodes qui se sont appliquées, les techniques alternatives de règlements des conflits, la gestion d'instance, les problèmes causés par la montée du phénomène de l'auto-représentation des plaideurs commandent une autre attitude. Celle-ci exige une volonté de culture, de conscience et d'attention à l'environnement social, la capacité de critique vis-à-vis de soi et des autres, le désir de faire et non pas de laisser faire. Les juges ne sont pas des activistes sociaux. Ils n'ont pas de thèses à défendre. Cependant, l'efficacité de la justice leur interdit l'ignorance et la passivité. Elle exige d'eux l'exécution d'un devoir impérieux de culture. L'éthique de la distance du monde n'est pas celle de l'ignorance de celui-ci. 